\title{
Calcium scoring using virtual non-contrast images from a dual-layer spectral detector CT: comparison to true non-contrast data and evaluation of proportionality factor in a large patient collective
}

\author{
Felix G. Gassert ${ }^{1}$ (D) . Claudio E. Schacky ${ }^{1} \cdot$ Christina Müller-Leisse $^{1}$ • Florian T. Gassert ${ }^{1} \cdot$ Gregor Pahn $^{2}$ • \\ Karl-Ludwig Laugwitz ${ }^{3}$ - Marcus R. Makowski ${ }^{1}$. Jonathan Nadjiri ${ }^{1}$
}

Received: 22 June 2020 / Revised: 23 November 2020 / Accepted: 29 December 2020 / Published online: 20 January 2021

(C) The Author(s) 2021

\begin{abstract}
Objective Determination of coronary artery calcium scoring (CACS) in non-contrast computed tomography (CT) images has been shown to be an important prognostic factor in coronary artery disease (CAD). The objective of this study was to evaluate the accuracy of CACS from virtual non-contrast (VNC) imaging generated from spectral data in comparison to standard (true) noncontrast (TNC) imaging in a representative patient cohort with clinically approved software.

Methods One hundred three patients referred to coronary CTA with suspicion of CAD were investigated on a dual-layer spectral detector CT (SDCT) scanner. CACS was calculated from both TNC and VNC images by software certified for medical use. Patients with a CACS of 0 were excluded from analysis.

Results The mean age of the study population was $61 \pm 11$ years with 48 male patients (67\%). Inter-quartile range of clinical CACS was 22-282. Correlation of measured CACS from true- and VNC images was high $(0.95) ; p<0.001$. The slope was 3.83, indicating an underestimation of VNC CACS compared to TNC CACS by that factor. Visual analysis of the Bland-Altman plot of CACS showed good accordance with both methods after correction of VNC CACS by the abovementioned factor.

Conclusions In clinical diagnostics of CAD, the determination of CACS is feasible using VNC images generated from spectral data obtained on a dual-layer spectral detector CT. When multiplied by a correction factor, results were in good agreement with the standard technique. This could enable radiation dose reductions by obviating the need for native scans typically used for CACS.

Key Points

- Calcium scoring is feasible from contrast-enhanced CT images using a dual-layer spectral detector CT scanner.

- When multiplied by a correction factor, calcium scoring from virtual non-contrast images shows good agreement with the standard technique.

- Omitting native scans for calcium scoring could enable radiation dose reduction.
\end{abstract}

Keywords Coronary artery disease $\cdot$ Vascular calcification $\cdot$ Computed tomography angiography

Felix G. Gassert

felix.gassert@tum.de

1 Department of Diagnostic and Interventional Radiology, School of Medicine \& Klinikum rechts der Isar, Technical University of Munich, Ismaningerstr. 22, 81675 Munich, Germany

2 Philips CT Clinical Science, Hamburg, Germany

3 Department of Internal Medicine I, School of Medicine \& Klinikum rechts der Isar, Technical University of Munich, Munich, Germany

\section{Abbreviations}

CACS Coronary artery calcium scoring

CAD Coronary artery disease

CT Computed tomography

CTA Computed tomography angiography

CTDI Computed tomography dose index

DLP Dose length product

HU Hounsfield units

SDCT Spectral detector CT

TNC True non-contrast

VNC Virtual non-contrast 


\section{Introduction}

Ischemic heart disease is the most common global cause of death, with high mortality reported in the developed countries [1]. Therefore, early detection of coronary artery disease (CAD) is crucial for the prevention of adverse events through dedicated therapy. Hence, non-invasive cardiac imaging by computed tomography plays an increasingly significant role in diagnosis of CAD [2-4]. Besides contrast-enhanced imaging for the evaluation of vascular stenosis, several studies showed that the determination of coronary artery calcium scoring (CACS) is an essential prognostic factor and a strong and independent predictor of cardiovascular events, such as myocardial infarction and sudden cardiac death $[3,5,6]$. The most commonly used method to evaluate patients' burden of $\mathrm{CAD}$ is the Agatston score, which measures the amount of calcium present in each lesion scaled by an attenuation factor and summed over all lesions [7].

Whereas currently both contrast-enhanced CT imaging for stenosis determination and non-contrast images for CACS are widely established for the diagnosis of CAD, several methods using spectral imaging including the possibility to generate virtual non-contrast (VNC) images for calculation of CACS have previously been proposed in multiple studies [8-10]. Dual-energy imaging is based on simultaneous acquisition of two CT datasets at different x-ray spectra — either by data acquisition at two different $\mathrm{x}$-ray tube voltages (dual-source $\mathrm{CT}, \mathrm{kV}$ switching) or by energy separation in the detector (dual-layer CT). Based on decomposition into two base materials (soft tissue and iodine), a virtual non-contrast image can be generated from a contrast-enhanced image [11, 12]. Especially the novel technique of spectral CT imaging using a dual-layer spectral detector CT (SDCT) system might help to overcome some limitations of other dual-energy techniques. For example, there is no temporal or projection offset using the dual-layer method. Furthermore, the dual-layer method has been reported to be a reliable method of dual-energy imaging $[13,14]$.

In a preliminary study, Nadjiri et al showed that the use of spectral imaging with SDCT for the determination of CACS from contrast-enhanced coronary computed tomography angiography (coronary CTA) may be a feasible alternative and reaches good agreement with the conventional technique. Nevertheless, the results of CACS (Agatston score) from VNC images needed to be multiplied by a proportionality factor of 1.83 to match the results from TNC images due to underestimation of plaque density and plaque volume with the VNC data. Furthermore, the sample size was small and patients without coronary calcifications were included in statistical analysis, resulting in a higher correlation [5].

The use of VNC images generated from spectral data could reduce radiation exposure by omitting additional native scans when performing a CTA. For establishing this method in everyday clinical practice, we sought to evaluate a more representative patient cohort and compare VNC with true noncontrast imaging. Furthermore, this study analyzes the extent and causes of needed proportionality factors.

\section{Material and methods}

\section{Study population}

Approval of the Institutional Review Board had been obtained prior to this study. Written informed consent was waived for this retrospective analysis of routinely acquired imaging and clinical data. All patients who underwent CACS and coronary CTA due to suspected CAD using spectral CT at our institution were eligible for the study. All patients without calcified plaques in true non-contrast (TNC) images (corresponding to a calcium scoring of 0 ) were excluded from statistical analysis.

\section{Computed tomography scans}

Heart rates lower than $60 \mathrm{bpm}$ were preferred for all examinations. Therefore, in patients with heart rates above $60 \mathrm{bpm}$, we administered up to $20 \mathrm{mg}$ metoprolol intravenously before scanning. Additionally, $0.8 \mathrm{mg}$ nitroglycerin was administered just before scanning for vasodilatation of coronary arteries when systolic blood pressure was above $100 \mathrm{mmHg}$.

A 64-slice single-source dual-layer spectral CT system was used for imaging (IQon; Philips Healthcare). The firmware used on the scanner was Version 4.7.0. A tube voltage of $120 \mathrm{kVp}$ was applied for both native and contrast-enhanced scans. Tube current time product for CACS was $35 \mathrm{mAs}$ and $140 \mathrm{mAs}$ for coronary CTA. Reconstruction of native scans was performed with a XCB kernel and reconstructions of coronary with $\mathrm{CB}$ (both standard cardiac kernels). Both reconstructions had identical spatial resolution and slice thickness.

ECG-triggered sequential scans were used for the acquisition of non-contrast images (as reference) and contrastenhanced images. Bolus tracking was applied for the timing of the contrast phase in contrast-enhanced images. In total, $80 \mathrm{ml}$ of contrast agent (Ultravist 370, Bayer, Bayer AG, iodine content $370 \mathrm{mg} / \mathrm{ml}$ ) was used for the contrastenhanced scans, applying a flow rate of $4-6 \mathrm{ml} / \mathrm{s}$ followed by a $50-\mathrm{ml}$ bolus of saline chaser.

\section{Post processing}

VNC images were generated from contrast-enhanced images using a software package which is certified for medical use and commercially available (IntelliSpace Portal version 10, Philips Healthcare). A slice thickness of $2 \mathrm{~mm}$ was used for both TNC and VNC images. 
Calcium score from both TNC and VNC was determined (IntelliSpace Portal). The threshold for plaque inclusion was set to 130 Hounsfield units (HU) as previously described in the literature and clinically used [7, 13, 15, 16]. All intraluminal (coronary arteries) plaques were selected and used for calcium scoring. Results using TNC and VNC data were compared.

\section{Statistical analysis}

All statistical analyses were performed using the statistical package $\mathrm{R}$ version 3.2.4 ( $\mathrm{R}$ Foundation for Statistical Computing). Categorical variables are expressed as frequencies and percentages, continuous variables are expressed as mean \pm standard deviation. For analysis of method agreement, Bland-Altman plots were applied. Method of least squares was used for linear regression between methods. Pearson's correlation coefficient was calculated for measuring the association between variables. A $p$ value $<0.05$ was considered statistically significant. $G^{*}$ Power was used for the analysis of post hoc study power.

\section{Results}

\section{Study population and radiation dose}

One hundred three consecutive patients with suspected CAD were scanned using the dual-layer SDCT system between January 2018 and March 2020. Fifty-five patients $(53.4 \%)$ were referred to coronary CTA for atypical cardiac symptoms, 26 patients $(25.2 \%)$ for typical cardiac symptoms, and $22(21.4 \%)$ for evaluation of operability. Out of the study population, 32 patients $(31.07 \%)$ were diagnosed with obstructive coronary artery disease. Thirty-one patients were excluded from primary statistical analysis due to calcium scoring of 0 as determined by the TNC images. Twenty-three of these patients had a CACS of 0 , three of these patients had a CACS of 1 , four had a CACS of 2, and one had a CACS of 4 using the VNC data. Forty-eight of the remaining 72 patients were male (66.7 \%). The mean age was $61.3 \pm 10.8$ years (IQR: 54.4-69.0 years; range: $36-85$ years).

For non-contrast-enhanced scans, computed tomography dose index (CTDI) was $4.15 \pm 0.29$ and dose length product (DLP) was $64.32 \pm 9.43$ resulting in an estimated effective dose of $1.15 \pm 0.17 \mathrm{mSv}$. For contrast-enhanced scans, CTDI was $17.01 \pm 2.90$ and DLP was $266.6 \pm 60.82$ resulting in a total effective dose of $4.81 \pm 1.09 \mathrm{mSv}$. The average time between non-contrast and contrast-enhanced scans was $4.63 \mathrm{~min}$.

\section{Comparison between calcium scoring from true and virtual non-contrast images}

The use of TNC resulted in a mean calcium score of 178 with a minimum value of 1 , a maximum value of 2048 , and an inter-quartile range of 22-282. Calculations of calcium scoring using VNC spectral images of the same patients resulted in a mean calcium score of 43 with a minimum value of 0 , a maximum value of 518, and an inter-quartile range of 7-56. Only one patient with a CACS of 7 on TNC images was wrongly classified as having a CACS of 0 on VNC images. Figure 1 shows a representative example of TNC, contrastenhanced, and VNC images of a patient.

There was a very high and significant correlation of calcium scoring calculated from TNC and VNC images $(0.95)(p<$ 0.001 ) and an acquired power of $100 \%$ for this result. Overall difference of means between methods was 4.14-fold. For the determination of exact proportionality factor, linear regression using the method of least squares was applied (Fig. 2). The slope was 3.83 indicating an underestimation of calcium scores obtained using the VNC data compared to those obtained from the TNC data by that factor. The intercept was 3.8 indicating a correct association between methods.

Using the slope derived from the linear regression model as proportionality constant, visual analysis of the Bland-Altman plot was performed. When the results from the VNC data are multiplied by this slope, CACS shows good accordance between both methods as shown in Fig. 3. Only 3 of 72 cases $(4.2 \%)$ are located outside the \pm 1.96 standard deviation range of difference. Regarding the grading of coronary artery disease (low risk: $\mathrm{CACS}=1-100$, moderate risk: $\mathrm{CACS}=101-$ 400, high risk: CACS > 400), 60 patients $(83.3 \%)$ were assigned to the same category, 4 in the category above, and 8 in the category below using VNC data as compared to TNC data.

\section{Discussion}

The determination of the coronary artery calcium score is an essential prognostic factor in patients with symptoms of coronary artery disease. Therefore, it is common practice to perform non-contrast-enhanced, electrocardiogram-gated scans for calcium quantification prior to CT angiography [17, 18]. Finding an approach for the determination of calcium score from contrast-enhanced images and thus omitting native scans preceding $\mathrm{CT}$ angiography would be useful for radiation dose reduction and shortening of the duration of the overall exam.

In this study including 103 patients with a correlation coefficient of 0.95 , we showed that there is a very high correlation between results of calcium scoring from real non-contrast images as currently performed in clinical routine and VNC images derived from contrast-enhanced imaging of a 64- 


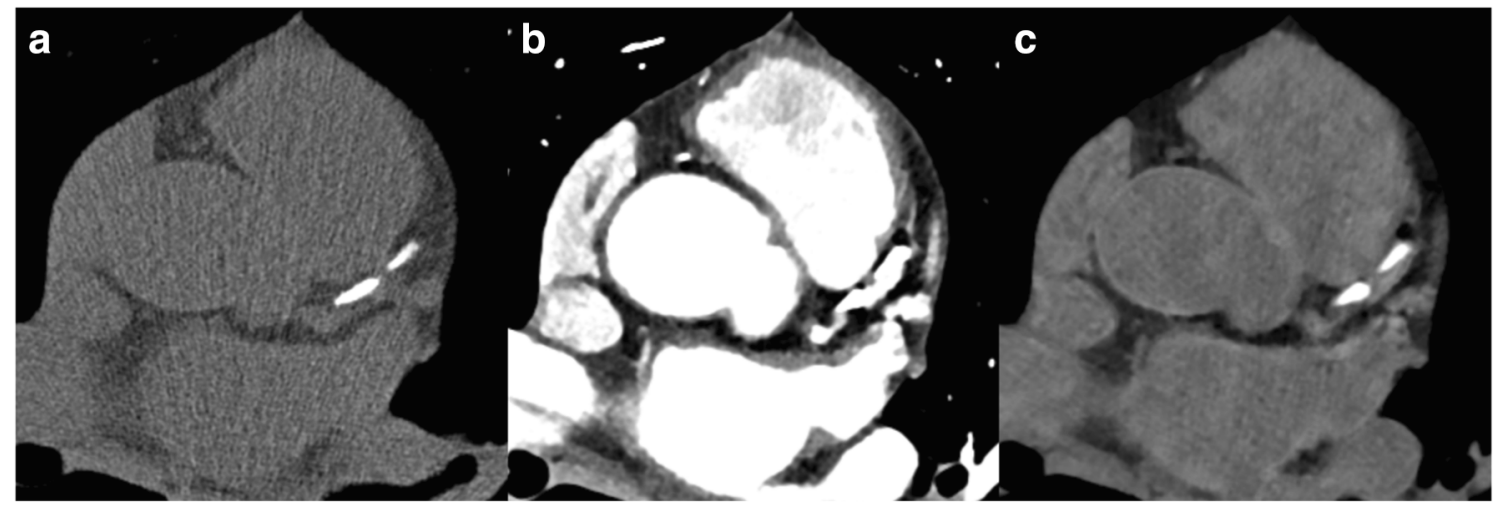

Fig. 1 True non-contrast image from a native scan (a), contrast-enhanced image (b), and virtual non-contrast image (c) from a coronary computed tomography angiography (coronary CTA)

slice single-source dual-layer spectral CT system through a software package certified for medical use and commercially available.

These results in general are in line with previous studies which also have shown good results of calculation of calcium score from VNC images. Schwarz et al demonstrated a correlation coefficient of 0.95 in 36 patients in a dual-source CT system and Fuchs et al showed a high agreement between methods with a correlation coefficient of 0.96 in 52 patients using a rapid $\mathrm{kVp}$-switching system and applying a reduced dose of contrast media $[8,9]$. Nevertheless, this is the first study to evaluate the accuracy of CACS from VNC imaging computed from spectral data on a SDCT scanner in comparison to standard non-contrast imaging in a more representative patient cohort with clinically approved software.
Additionally, it is of note that contrary to other studies, patients with a CACS of zero were excluded in our study to better understand the correlation of the measured values $[8,9]$. However, the intercept was 3.8 and therefore close to zero, indicating the correct association between the methods. Furthermore, of the 31 patients with CACS of zero, none exhibited virtual CACS of $\geq 5$ indicating a low rate of falsepositive results. The false-positive result was visually assessed and seems to be most likely caused by reconstruction artefacts as in very few occasions wall adherent contrast agent was not fully subtracted from the image.

Despite the exclusion of patients with a calcium score of zero, Pearson's correlation coefficient of 0.95 still indicates one of the highest correlations of calcium scoring values between true and virtual non-contrast images and $83.3 \%$ of
Fig. 2 Correlation between calcium scoring using true and virtual non-contrast images. RCACS, calcium scoring using true non-contrast images; VCACS, calcium scoring using virtual non-contrast images

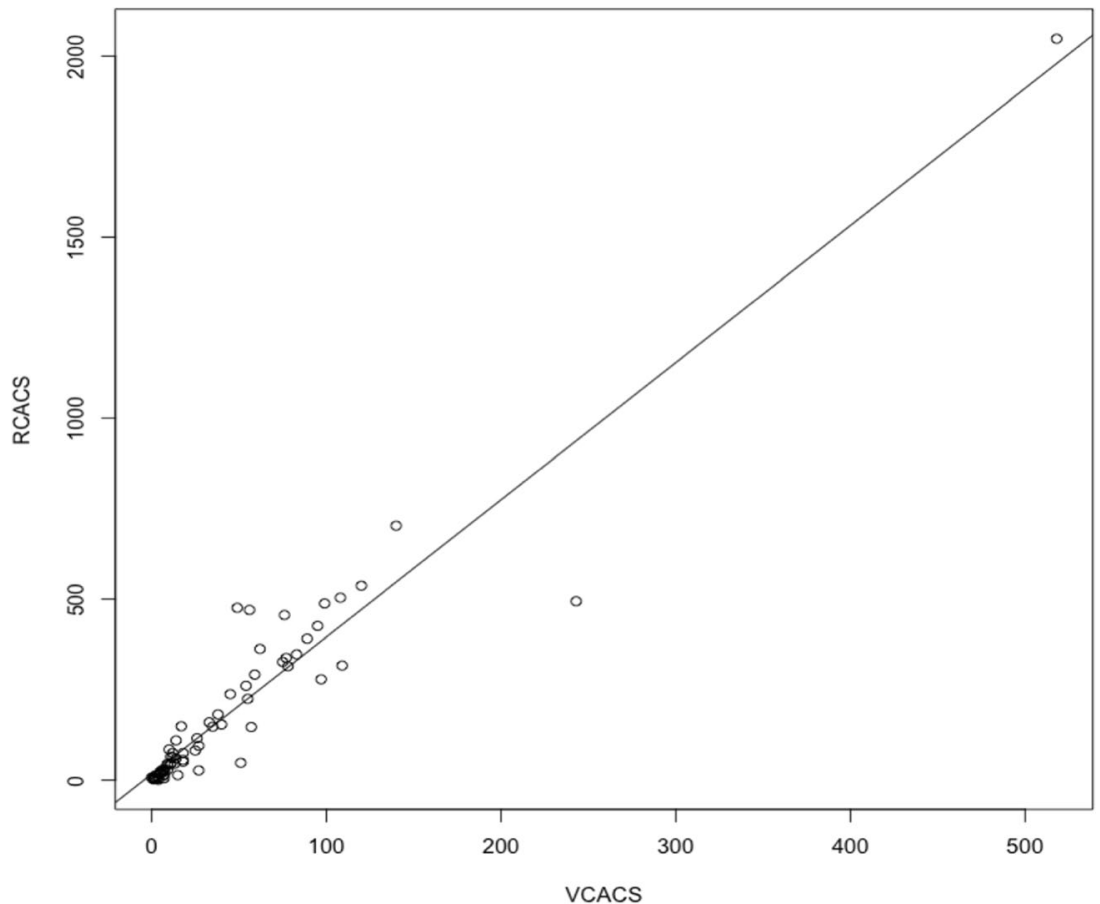


Fig. 3 The Bland-Altman plot for the ratio between calcium scoring using true and virtual noncontrast images

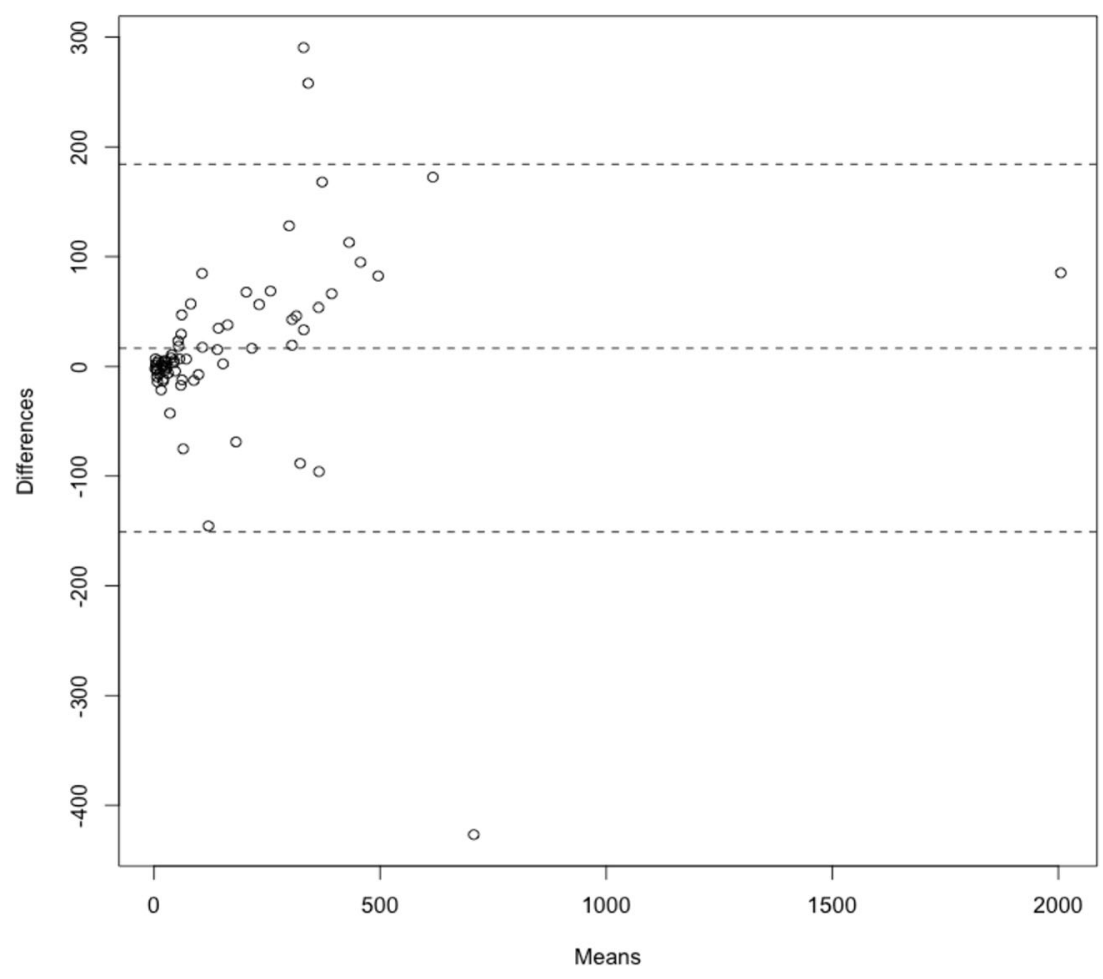

patients were classified in the same category comparing CACS calculated from TNC and VNC $[8,9,19,20]$.

Nevertheless, there is still a small residual discrepancy with $16.7 \%$ of patients being classified in a higher or lower risk category when using VNC as compared to TNC. This discrepancy does not necessarily need to be attributed to the difference in methods as a previous study showed a notable interscan variability within 5 min using the same CT equipment [21]. Additionally, different standard kernels used and processing through dedicated software might further influence the outcome of CACS [22].

For proper transfer of results between methods, a proportionality factor needs to be applied as analysis of real noncontrast images showed a calcium score 3.83 times as high as calcium scores analyzed in VNC images. As virtual noncontrast is based on decomposition into two different materials (soft tissue and iodine), attenuation of calcium can be reduced as a consequence. In a study published recently, Nadjiri et al showed that there might be two reasons for the underestimation of calcium scoring in VNC data: a slight underestimation of the plaque volume and, more importantly, an underestimation of plaque attenuation in VNC images; a reduced attenuation of Ca-plaques is to expect in the $\mathrm{VNC}$ reconstruction. Therefore, certain plaques will be excluded from the semiautomatic analysis as they will not exceed the threshold of $130 \mathrm{HU}$ in the VNC reconstruction [5].

The goal of this study was to show a way of reducing radiation in analysis of CACS using a dual-layer SDCT.
Tube-based dual-energy CT systems have been reported to increase radiation exposure depending on the patient's heart rate, protocol, and scanner generation [23, 24]. Nevertheless, improved conventional detectors with higher dose efficiency as well as the omission of native scans reduced overall radiation exposure compared to current clinical standard procedures in CT-based evaluation of coronary artery disease by approximately $20-25 \%[25,26]$. When omitting the native scan in this study, effective dose could be reduced by $19.3 \%$. The effective dose of $4.81 \mathrm{mSv}$ for the contrastenhanced scan with our scanner is comparable to radiation doses commonly published in the literature for this type of examination, although lower effective doses can be achieved when using e.g. 320-detector-row single-source CT scanners or dual-source CT scanners [27, 28].

Despite its clinical setting, high number of subjects, and good correlation, our study has several limitations. First of all, CT-based true non-contrast imaging was used as a reference standard. Although beam hardening and blooming have been described in the context of calcified coronary artery lesions and could lead to artifacts, they could also occur in native scans $[8,29]$. Nevertheless, there is no other feasible method available in a clinical setting.

There are some differences in our results from this study compared to previously published findings, where Nadjiri et al reported a proportionality factor of 1.83 . Some of these differences could be attributed to difference in sample size, exclusion of patients with CACS of 
zero, and different software and conversion tool used. Additionally, Nadjiri et al used a threshold of $90 \mathrm{HU}$ for CACS whereas, in this study, the threshold was set to $130 \mathrm{HU}$ as commonly used for Agatston scoring [5, 7]. For transfer into daily clinical use, confirmation of results in a multi-center study and precise definition of extent and causes of proportionality factor is needed.

To conclude, this is the first study to demonstrate that the determination of coronary artery calcium score is feasible using VNC images obtained from a dual-layer spectral detector $\mathrm{CT}$ in a representative patient cohort. By applying a correction factor, the results show good agreement with the standard technique. This could obviate the need for native scans typically used for CACS, thereby facilitating radiation dose reductions. Nevertheless, translation into clinical practice is subject to further studies and evaluation of causes of needed proportionality factor.

Funding Open Access funding enabled and organized by Projekt DEAL. The authors state that this work has not received any funding.

\section{Compliance with ethical standards}

Guarantor The scientific guarantor of this publication is Jonathan Nadjiri.

Conflict of interest The authors of this manuscript declare relationships with the following companies:

Gregor Pahn: Employee at Philips CT Clinical Science, Hamburg, Germany.

All other authors of this manuscript declare no relationships with any companies whose products or services may be related to the subject matter of the article.

Statistics and biometry No complex statistical methods were necessary for this paper.

Informed consent Written informed consent was waived by the Institutional Review Board.

Ethical approval Institutional Review Board approval was obtained.

Methodology
- retrospective
- diagnostic or prognostic study
- performed at one institution

Open Access This article is licensed under a Creative Commons Attribution 4.0 International License, which permits use, sharing, adaptation, distribution and reproduction in any medium or format, as long as you give appropriate credit to the original author(s) and the source, provide a link to the Creative Commons licence, and indicate if changes were made. The images or other third party material in this article are included in the article's Creative Commons licence, unless indicated otherwise in a credit line to the material. If material is not included in the article's Creative Commons licence and your intended use is not permitted by statutory regulation or exceeds the permitted use, you will need to obtain permission directly from the copyright holder. To view a copy of this licence, visit http://creativecommons.org/licenses/by/4.0/.

\section{References}

1. Nowbar AN, Gitto M, Howard JP, Francis DP, Al-Lamee R (2019) Mortality from ischemic heart disease. Circ Cardiovasc Qual Outcomes 12:e05375

2. Sanchis-Gomar F, Perez-Quilis C, Leischik R, Lucia A (2016) Epidemiology of coronary heart disease and acute coronary syndrome. Ann Transl Med 4:256

3. Budoff MJ, Shaw LJ, Liu ST et al (2007) Long-term prognosis associated with coronary calcification: observations from a registry of 25,253 patients. J Am Coll Cardiol 49:1860-1870

4. Han D, Lee JH, Hartaigh B, Min JK (2016) Role of computed tomography screening for detection of coronary artery disease. Clin Imaging 40:307-310

5. Nadjiri J, Kaissis G, Meurer F et al (2018) Accuracy of calcium scoring calculated from contrast-enhanced coronary computed tomography angiography using a dual-layer spectral CT: a comparison of calcium scoring from real and virtual non-contrast data. PLoS One 13:e0208588

6. Uehara M, Takaoka H, Kobayashi Y, Funabashi N (2013) Diagnostic accuracy of 320-slice computed-tomography for detection of significant coronary artery stenosis in patients with various heart rates and heart rhythms compared with conventional coronary-angiography. Int J Cardiol 167:809-815

7. Agatston AS, Janowitz WR, Hildner FJ, Zusmer NR, Viamonte M Jr, Detrano R (1990) Quantification of coronary artery calcium using ultrafast computed tomography. J Am Coll Cardiol 15:827-832

8. Schwarz F, Nance JW Jr, Ruzsics B, Bastarrika G, Sterzik A, Schoepf UJ (2012) Quantification of coronary artery calcium on the basis of dual-energy coronary CT angiography. Radiology 264:700-707

9. Fuchs TA, Stehli J, Dougoud S et al (2014) Coronary artery calcium quantification from contrast enhanced $\mathrm{CT}$ using gemstone spectral imaging and material decomposition. Int J Cardiovasc Imaging 30: 1399-1405

10. Yamada Y, Jinzaki M, Okamura T et al (2014) Feasibility of coronary artery calcium scoring on virtual unenhanced images derived from single-source fast $\mathrm{kVp}$-switching dual-energy coronary $\mathrm{CT}$ angiography. J Cardiovasc Comput Tomogr 8:391-400

11. Goo HW, Goo JM (2017) Dual-Energy CT: New horizon in medical imaging. Korean J Radiol 18:555-569

12. Schoepf UJ, Colletti PM (2012) New dimensions in imaging: the awakening of dual-energy CT. AJR Am J Roentgenol 199:S1-S2

13. McCollough CH, Leng S, Yu L, Fletcher JG (2015) Dual- and multi-energy CT: principles, technical approaches, and clinical applications. Radiology 276:637-653

14. Altman A, Carmi R (2009) TU-E-210A-03: A double-layer detector, dual-energy ct - principles, advantages and applications. Med Phys 36:2750-2750

15. Yoon HC, Greaser LE 3rd, Mather R, Sinha S, McNitt-Gray MF, Goldin JG (1997) Coronary artery calcium: alternate methods for accurate and reproducible quantitation. Acad Radiol 4:666-673

16. Callister TQ, Cooil B, Raya SP, Lippolis NJ, Russo DJ, Raggi P (1998) Coronary artery disease: improved reproducibility of calcium scoring with an electron-beam CT volumetric method. Radiology 208:807-814

17. Leschka S, Scheffel H, Desbiolles L et al (2008) Combining dualsource computed tomography coronary angiography and calcium scoring: added value for the assessment of coronary artery disease. Heart 94:1154-1161

18. Simon J, Szaraz L, Szilveszter B et al (2020) Calcium scoring: a personalized probability assessment predicts the need for additional or alternative testing to coronary CT angiography. Eur Radiol. https://doi.org/10.1007/s00330-020-06921-7 
19. Song I, Yi JG, Park JH, Kim SM, Lee KS, Chung MJ (2016) Virtual non-contrast CT using dual-energy spectral ct: feasibility of coronary artery calcium scoring. Korean J Radiol 17:321-329

20. Mahoney R, Pavitt CW, Gordon D et al (2014) Clinical validation of dual-source dual-energy computed tomography (DECT) for coronary and valve imaging in patients undergoing trans-catheter aortic valve implantation (TAVI). Clin Radiol 69:786-794

21. Hokanson JE, MacKenzie T, Kinney G et al (2004) Evaluating changes in coronary artery calcium: an analytic method that accounts for interscan variability. AJR Am J Roentgenol 182:1327-1332

22. Ghadri JR, Goetti R, Fiechter M et al (2011) Inter-scan variability of coronary artery calcium scoring assessed on 64-multidetector computed tomography vs. dual-source computed tomography: a headto-head comparison. Eur Heart J 32:1865-1874

23. Rixe J, Conradi G, Rolf A et al (2009) Radiation dose exposure of computed tomography coronary angiography: comparison of dualsource, 16-slice and 64-slice CT. Heart 95:1337-1342

24. Kirchhoff S, Herzog P, Johnson T et al (2010) Assessment of radiation exposure on a dual-source computed tomography-scanner performing coronary computed tomography-angiography. Eur $\mathrm{J}$ Radiol 74:e181-e185
25. Wichmann JL, Hardie AD, Schoepf UJ et al (2017) Single- and dual-energy CT of the abdomen: comparison of radiation dose and image quality of 2 nd and 3rd generation dual-source CT. Eur Radiol 27:642-650

26. Jin L, Gao Y, Jiang A, Li Z, Wang P, Li M (2020) Can the coronary artery calcium score scan reduce the radiation dose in coronary computed tomography angiography? Acad Radiol. https://doi.org/ 10.1016/j.acra.2020.02.014

27. Tan SK, Yeong CH, Ng KH, Abdul Aziz YF, Sun Z (2016) Recent update on radiation dose assessment for the state-of-the-art coronary computed tomography angiography protocols. PLoS One 11: e 0161543

28. Machida H, Tanaka I, Fukui R et al (2015) Current and novel imaging techniques in coronary CT. Radiographics 35:991-1010

29. Maass C, Baer M, Kachelriess M (2009) Image-based dual energy CT using optimized precorrection functions: a practical new approach of material decomposition in image domain. Med Phys 36: $3818-3829$

Publisher's note Springer Nature remains neutral with regard to jurisdictional claims in published maps and institutional affiliations. 